\title{
Design and testing of hydrophobic core/ hydrophilic shell nano/micro particles for drug-eluting stent coating
}

\author{
Ruolin Du', Yazhou Wang', Yuhua Huang', Yinping Zhao', Dechuan Zhang ${ }^{2}$, Dingyuan Du², Yuan Zhang ${ }^{3}$, \\ Zhenggong $\mathrm{Li}^{3}$, Sean McGinty ${ }^{4}$, Giuseppe Pontrelli ${ }^{5}$, Tieying Yin ${ }^{1}$ and Guixue Wang ${ }^{1}$
}

\begin{abstract}
In this study, we designed a novel drug-eluting coating for vascular implants consisting of a core coating of the antiproliferative drug docetaxel (DTX) and a shell coating of the platelet glycoprotein IIb/llla receptor monoclonal antibody SZ-21. The core/shell structure was sprayed onto the surface of $316 \mathrm{~L}$ stainless steel stents using a coaxial electrospray process with the aim of creating a coating that exhibited a differential release of the two drugs. The prepared stents displayed a uniform coating consisting of nano/micro particles. In vitro drug release experiments were performed, and we demonstrated that a biphasic mathematical model was capable of capturing the data, indicating that the release of the two drugs conformed to a diffusion-controlled release system. We demonstrated that our coating was capable of inhibiting the adhesion and activation of platelets, as well as the proliferation and migration of smooth muscle cells (SMCs), indicating its good biocompatibility and anti-proliferation qualities. In an in vivo porcine coronary artery model, the SZ-21/DTX drug-loaded hydrophobic core/hydrophilic shell particle coating stents were observed to promote re-endothelialization and inhibit neointimal hyperplasia. This core/shell particle-coated stent may serve as part of a new strategy for the differential release of different functional drugs to sequentially target thrombosis and in-stent restenosis during the vascular repair process and ensure rapid re-endothelialization in the field of cardiovascular disease.
\end{abstract}

\section{Introduction}

Cardiovascular diseases are among the most common diseases that give rise to disability and mortality. Currently, the use of coronary stents is commonplace in the treatment of such diseases ${ }^{1}$. Extensive data from experimental and clinical studies confirm that although drugeluting stents (DESs) reduce the rate of restenosis compared with bare-metal stents (BMSs), there remains an

\footnotetext{
Correspondence: Guixue Wang (wanggx@cqu.edu.cn) or

Tieying Yin (ilyyty28@126.com)

${ }^{1}$ Key Laboratory for Biorheological Science and Technology of Ministry of Education, State and Local Joint Engineering Laboratory for Vascular Implants, Bioengineering College of Chongqing University, Chongqing 400030, China ${ }^{2}$ The Affiliated Central Hospital of Chongqing University and Chongqing Emergency Medical Center, Chongqing 400014, China Full list of author information is available at the end of the article. These authors contributed equally: Ruolin Du, Yazhou Wang.
}

increased risk of late stent thrombosis with $\mathrm{DESs}^{2-6}$. Percutaneous coronary intervention (PCI), especially involving stents, usually results in injury to the endothelium, consequently inducing the activation and gathering of blood platelets to form thrombosis, and the stent may become a site for the adhesion of platelets before they are completely covered by neointima ${ }^{4,7}$. Other long-term clinical studies have reported that DESs loaded with antiproliferative agents suppressed the growth of neointima, delayed the re-endothelialization of stents, lengthened the time for activation of platelets, and increased the risk of late thrombosis ${ }^{8}$. Hence, the design of DESs in the future must consider these critical issues.

The anti-proliferative drugs rapamycin (also known as sirolimus) and paclitaxel were the first-generation drugs coated with DES approved by the US FDA. Subsequently, 
as newer-generation stents have emerged, sirolimus analogues such as zotarolimus and everolimus have been utilized. Despite these advancements, today's DES have not completely eradicated restenosis and still lead to a higher incidence of late stent thrombosis. Drug docetaxel (DTX) is a paclitaxel analogue with enhanced anti-proliferative ability and water solubility and has been shown to be a good candidate drug for the prevention of restenosis ${ }^{9,10}$. Thrombosis remains a concern after stent implantation, whether at the early or very late stage. The exposure of damaged endothelial cells to the blood activates platelet adhesion and aggregation. The binding of the monoclonal antibody (mAb) to the platelet GP IIb/IIIa receptor ${ }^{11}$ has potent anti-platelet and anti-thrombotic characteristics that have been shown to reduce thrombosis-related major complications after coronary angioplasty ${ }^{12-14}$.

Polymer particles can be employed to deliver medication in a rate-controlled and sometimes targeted manner by drug leaching from the polymer or by degradation of the polymer matrix ${ }^{15}$. The advancement and convergence of particle drug delivery systems have recently been explored to optimize stent coatings. For example, drugs exhibiting different therapeutic effects were encapsulated in bi-layered PLGA particles containing vascular endothelial growth factor (VEGF) plasmid and paclitaxel; stents with this particle coating promoted early endothelium healing and inhibited smooth muscle cell (SMC) proliferation $^{16-18}$. Local particle-based drug delivery was hypothesized to facilitate high regional concentrations of therapeutic agents with prolonged retention at low doses, leading to reduced systemic toxicity ${ }^{19,20}$. For drug loading and release of particles, there already exist several approaches ${ }^{21}$. Traditional particle preparation methods cannot adequately control the burst release of drugs ${ }^{22}$. Electrospraying, on the other hand, provides a superior alternative to producing stent coatings with different nano/micro particle sizes and assembly methods. Thian et $\mathrm{al}^{23}$ used electrospraying deposition to produce a uniform coating consisting of hydroxyapatite nanocrystals on a titanium substrate, which triggered an early apatite precipitation process in cellular-simulated body fluid. Almería et $\mathrm{al}^{24}$ demonstrated a multiplexed electrospraying process for the single-step synthesis of stabilized polymer particles for drug delivery, but the release rate of the drugs was found to be rapid, reaching approximately $70 \%$ within the first $4 \mathrm{~h}$. To the best of our knowledge, only a limited number of studies have reported the application of electrospraying for the fabrication of vascular DES coatings. In particular, Dong et al. fabricated and controlled the release of the electrosprayed ReoProloaded metal vascular stent. Hydrophilic ReoPro release was controlled in the hydrophobic PLGA-based release system and was effective in suppressing platelet adhesion and SMC overgrowth for vascular stents ${ }^{25}$. Zamani's research showed that multi-drug coatings could easily be fabricated using electrospraying. The electrosprayed Montelukast/poly(lactic-co-glycolic acid) particle-based coating is a potential new therapeutic approach toward the prevention of in-stent restenosis ${ }^{26}$.

Ideal vascular implant coatings should exhibit a number of properties. These properties include biocompatibility, anti-thrombogenicity, the ability to interface with antiproliferative drugs to reduce platelet activation level and time, restenosis prevention and a favorable surface topography for re-endothelialization ${ }^{27,28}$. To achieve dualfunctionalized vascular stents with dual drugs, researchers have chosen to combine various coating methods and multiple-step methods for coating preparation ${ }^{29,30}$. To the best of our knowledge, there is no published work that considers multi-hydrophilic/hydrophobic drug coating preparation for coronary stents with a single step. In our previous research, we reported the detection of sequential drug release of polymer-controlled core/shell nanoparticles prepared by coaxial electrospraying ${ }^{31}$. Here, we designed a novel DES coating consisting of particles with a core of the anti-proliferative drug DTX and a shell of SZ-21, which was the platelet glycoprotein IIb/IIIa receptor monoclonal antibody ${ }^{32,33}$. The particles were produced and deposited on the stent by coaxial electrospraying (Fig. 1).

\section{Materials and methods \\ Materials}

Three hundred sixteen liters (316L) stainless steel (SS) stents $(3.0 \times 17 \mathrm{~mm})$ and $316 \mathrm{~L}$ SS sheets $(\varnothing 10 \times 1.5 \mathrm{~mm})$ were purchased from Beijing AmsinoMed Medical Device Co., Ltd., China. SZ-21 was supplied by Prof. Changgeng Ruan in the Jiangsu Institute of Hematology (First Affiliated Hospital of Suzhou University, Suzhou, China). Human umbilical vein endothelial cells (HUVECs) and Human umbilical arterial smooth muscle cells (HUASMCs) were a generous gift from Dr. Lushan Liu (Nan Hua University, China). DTX (Xieli Pharmaceutical Co., Ltd, Sichuan, China), chitosan (CS) $M_{w}=600,000$ (Boxin Biotech Co., Ltd, Tianjin, China) and poly [(50\% lactic acid) $(50 \%$ glycolic acid)] (PLGA) $M_{w}=20,000$ (Sigma Inc., St. Louis, USA) were also used in these studies.

\section{Multilayer coating with hydrophobic core/hydrophilic shell nano/micro particles by electrospraying}

Coating experiments were carried out on $316 \mathrm{~L}$ SS stents $(3.0 \times 17 \mathrm{~mm})$ and $316 \mathrm{~L}$ SS sheets $(\varnothing 10 \times 1.5 \mathrm{~mm})$ utilizing coaxial electrospraying. The design and experimental setup of the coating procedure are shown in Fig. 1.

Dichloromethane and ethylic acid solution were used as the solvents for PLGA and CS, respectively. In the electrospray coating process, $10 \mathrm{~mL}$ syringes containing DTX-PLGA solution (DTX concentration at $1 \mathrm{mg} / \mathrm{mL}$ ) or SZ-21-CS solution (SZ-21 concentration at $2 \mathrm{mg} / \mathrm{mL}$ ) were 


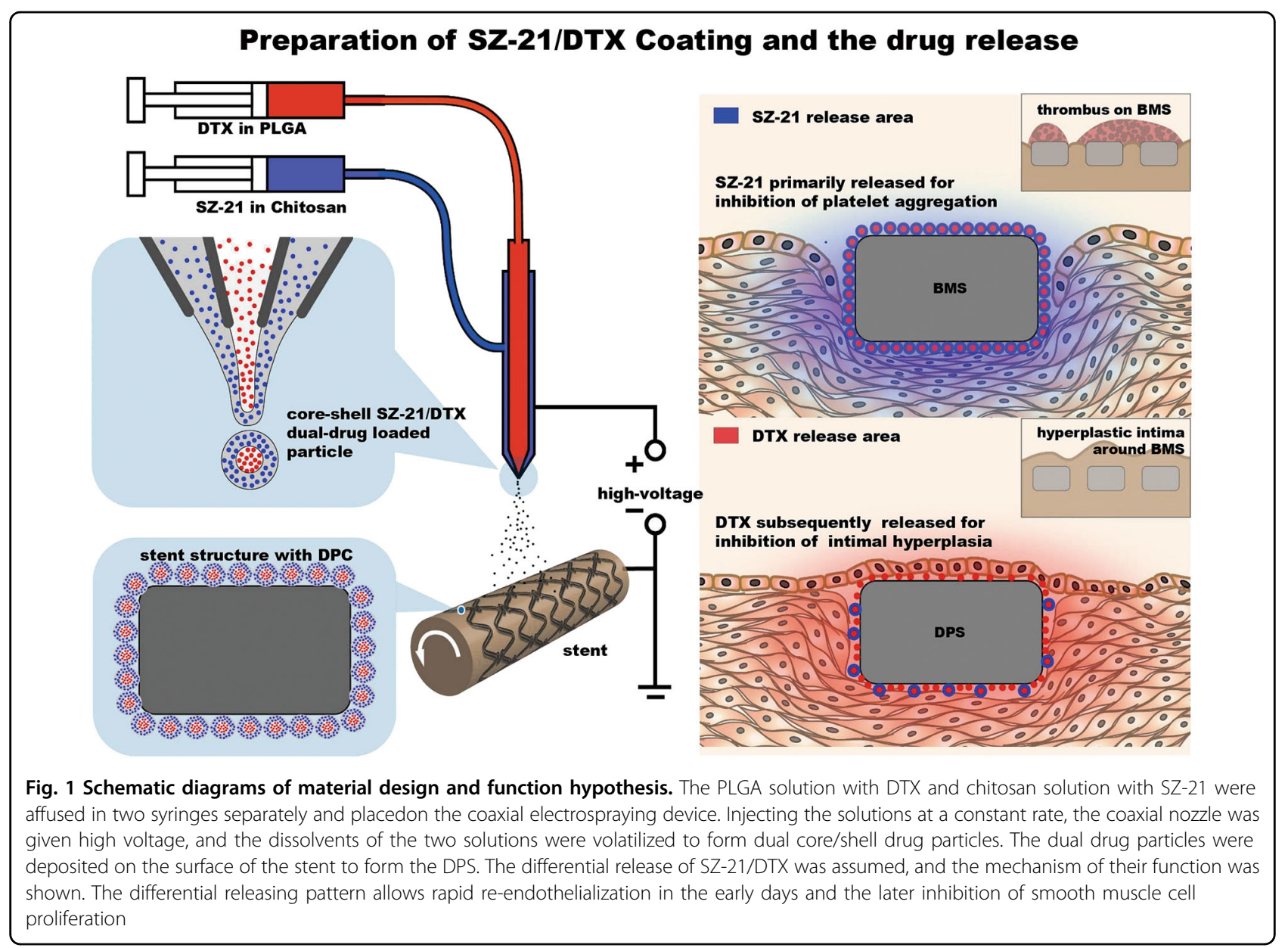

connected with a coaxial nozzle. The reception distance between the tip of the needle to the coating surface was maintained at $5-15 \mathrm{~cm}$; the voltage and inner/outer flow rate were changed to obtain a uniform coating (Table S1). The SZ-21/DTX drug-loaded hydrophobic core/hydrophilic shell particle coating (DPC) and no drug core/shell particle coating (BPC) were prepared according to the following selected parameters: $0.2 \mathrm{~mL} / \mathrm{h}$ (inner) and $0.6 \mathrm{~mL} / \mathrm{h}$ (outer), a PLGA concentration of $10 \mathrm{mg} / \mathrm{mL}$, a CS concentration of $2.5 \mathrm{mg} / \mathrm{mL}$, a reception distance of $5 \mathrm{~cm}$, and a voltage of $14 \mathrm{kV}$. The morphology, core/shell structure, and particle size distribution were detected through a fluorescence microscope (IX81, Olympus, Japan), scanning electron microscope (SEM; Vega-LMH, Tescan, Czech), confocal microscope (SP8, Leica, German) and transmission electron microscope (TEM, JEM-2100F, JEOL, Japan).

\section{Morphometric analysis of the SZ-21/DTX drug-loaded} hydrophobic core/hydrophilic shell particle coating stent (DPS)

\section{Fluorescence characterization of DPS}

DPS was prepared by coaxial electrospraying. To identify the hydrophobic core/hydrophilic shell particle nature of the coating, $1 \mathrm{mg} / \mathrm{mL}$ fluorescein isothiocyanate (FITC) was added to the shell solution and $1 \mathrm{mg} / \mathrm{mL}$ rhodamine $B$ was added to the core solution, allowing for observation by fluorescence microscope (IX81, Olympus, Japan).

\section{SEM and EDS test of DPS}

After coating, DPS was expanded three times for $30 \mathrm{~s}$ each time by balloon $(2.75 \mathrm{~mm} \times 18 \mathrm{~mm}$ MAVERJCK2, Boston scientific) at $10 \mathrm{~atm}$ in vitro; the changes in morphology and element contents of BMS, DPS and after expansion were all determined using a scanning electron microscope (SEM; Vega-LMH, Tescan, Czech) and energy dispersive spectrometer (EDS; INCA $\mathrm{x}$-sight 7557, Oxford Instruments, UK), respectively.

\section{Atomic force microscopy and water contact angle analysis of DPS}

The morphology changes of stents with different coatings were characterized using an atomic force microscope (AFM; SPI3800N, SEIKO, Japan). The hydrophilicity alteration of different coating surfaces, as determined by the distilled water contact angle, was measured with a contact angle tester (DSA30, Krüss, Germany) at room 
temperature. Three different sites were selected randomly for each group.

\section{Fourier transform infrared spectroscopy (FTIR) detection of drugs and polymers for DPS}

FTIR measurement was performed to analyze the chemical structure of the coaxial sprayed coatings before and after coaxial electrospraying. Using the squash method, $\mathrm{KBr}$ (100-200 mg) and solid samples (1-2 mg) were ground into micron powder and pressed into sheets $(\varnothing 13 \times 1.0 \mathrm{~mm})$ for analysis.

\section{Drug release analysis in vitro \\ Detection of drug release in vitro}

For drug release analysis, DPSs were transferred in $1 \mathrm{~mL}$ phosphate-buffered saline (PBS) in a constant temperature shaking incubator at $60 \mathrm{rpm} / \mathrm{min}$ and $37^{\circ} \mathrm{C}$. The concentrations of released drugs were monitored at pre-determined time points, and the fresh PBS was replaced each time. Moreover, the stent morphology after 0,7 , and 28 days was characterized by SEM. The SZ-21 release rate in vitro was tested using mice immunoglobulin IgG Elisa kits. DTX was assayed by HPLC (Agilent 1100, USA) with a C18 $250 \mathrm{~mm} \times 4.6 \mathrm{~mm}$ column. The mobile phase was acetonitrile: water (65:35). The sample was eluted at $1 \mathrm{~mL} / \mathrm{min}$ at room temperature, and the absorbance was measured at $230 \mathrm{~nm}$.

\section{Mathematical modeling of drug release in vitro}

The large number of core/shell particles on the coating made it impractical to mathematically model each individual particle. Instead, we adopted a continuum approach and approximated the core/shell coating layer as a biphasic material, providing two independent routes of transport for each drug. These two routes could result from surface preparation methods and may correspond to the drug phases being either fully embedded within the coating layer (slow release) or connected to the surface by pores or other defects (fast release) ${ }^{34}$.

Each route of transport was associated with a different effective transport speed characterized by a rate constant $k_{i}, i=1,2$. Assuming that the drug was initially uniformly distributed within each respective route, the strut was impermeable to drug transport, the release medium acted as an infinite sink and the drugs were dilute, and then considering drug transport within each route as a diffusion-dominated process, we derived an expression for the mass of each drug within the coating as a function of time, $M(t)$ :

$$
M(t)=\sum_{n=1}^{\infty} A(n)\left\{r e^{-K_{1}(n) t}+(1-r) e^{-K_{2}(n) t}\right\}
$$

where

$A(n)=\frac{8 M_{0}}{\pi^{2}(2 n-1)^{2}}, K_{1}(n)=\frac{(2 n-1)^{2} \pi^{2} k_{1}}{4}, K_{2}(n)=\frac{(2 n-1)^{2} \pi^{2} k_{2}}{4}$, and $r$ is the initial fraction of the total drug loading $\left(M_{0}\right)$ within each route.

Using the knowledge of the total drug loading for each drug and Eq. (1), we calculated the cumulative fraction of each drug that had been released as a function of time.

\section{Blood compatibility evaluation}

Blood was obtained from healthy volunteers in accordance with the protocol approved by the Blood Donation Law of the People's Republic of China. To isolate fresh human platelet-rich plasma (PRP), the blood was centrifuged at $1200 \mathrm{rpm}$ for $10 \mathrm{~min}$, and the supernatant was collected. All sheets were incubated in $0.5 \mathrm{~mL}$ of PRP for $30 \mathrm{~min}$ at $37^{\circ} \mathrm{C}$, rinsed with PBS, fixed with $2.5 \%$ glutaraldehyde for $12 \mathrm{~h}$ at $4{ }^{\circ} \mathrm{C}$ and dehydrated for $\mathrm{SEM}^{35}$. The expression of platelet granule membrane glycoprotein CD62P was detected to evaluate the anti-platelet activation of the coating. The deformability of adherent platelets (type I-V) on coatings was analyzed, and the morphological indices were calculated via the following formula: ${ }^{36}$

morphological index

$=\frac{(\text { No.type } I \times 1+\text { No.typeII } \times 2+\text { No.type } I I I \times 3+\text { No.type } I V \times 4+\text { No.type } V \times 5)}{\text { total } n u m b e r \text { f }}$ total number of adherent platelets

The hemolysis ratio of DPC was tested following the Chinese national standard (Biological Evaluation of Medical Devices-Part 4: Selection of Tests for Interactions with Blood, GB/T 16886.4-2003). The activated partial thrombin time (APTT) and prothrombin time (PT) were applied to evaluate the anti-thrombogenicity of stents in vitro ${ }^{37}$.

\section{The proliferation and migration of HUVEC and HUASMC on coatings \\ Cell culture}

HUVECs and HUASMCs were cultured in RPMI 1640 and DMEM/F12 media (HyClone, USA), respectively, supplemented with $10 \%$ fetal bovine serum (FBS; Cambrex Corp, USA) and 1\% P/S (Gibco Industries Inc, USA) at $37^{\circ} \mathrm{C}$ under $5 \% \mathrm{CO}_{2}$.

\section{HUVECs seeding and cell viability assay}

A rotation culture device after autoclave sterilization was used to seed cells on the stent surface. BMS, BPS, and DPS were transferred to the rotation culture tubes after undergoing ultraviolet irradiation sterilization. Then, HUVECs were seeded in the tubes at $5 \times 10^{4}$ cells/tube density with $10 \mathrm{rpm} / \mathrm{min}$ for $12 \mathrm{~h}$ at $37^{\circ} \mathrm{C}$. Finally, the stents were gently taken out in an aseptic condition and cultured in 12-well plates at $37^{\circ} \mathrm{C}$ under $5 \% \mathrm{CO}_{2}{ }^{38}$. 
After a 5-day culture, the stents were immobilized with $2.5 \%$ glutaraldehyde and dehydrated for morphological observation by SEM. The proliferation rates of cells on the stent surfaces were investigated with Cell Titer $96^{\circ}$ Aqueous One Solution Cell Proliferation Assay after 1, 3, and 5 days. The relative growth of rate (RGR) was calculated by this formula:

$$
R G R=\left(\frac{O D_{t}}{O D_{n c}}\right) \times 100 \%
$$

(where $\mathrm{OD}_{\mathrm{t}}$ represents the experimental group, and $\mathrm{OD}_{\mathrm{nc}}$ represents the negative control group).

After co-culturing for 1,3 , and 5 days, the culture supernatant was collected by centrifuging at $3000 \mathrm{rpm}$ for $10 \mathrm{~min}$. The nitric oxide (NO) release levels and total antioxidant capacity (T-AOC) of HUVECs were tested.

\section{The migration of HUVECS and HUASMCs on coatings}

HUVECs and HUASMCs were cultured separately. Both HUVEC and HUASMC migration assays were carried out with the classical scarification method. Different coatings were sprayed on 316 L SS sheets by coaxial electrospraying. Then, the sheets of three groups $(316 \mathrm{~L}$ SS, BPC, DPC) were placed in 24-well plates after undergoing ultraviolet irradiation sterilization for $12 \mathrm{~h}$. One milliliter of cell suspension $\left(1 \times 10^{5}\right.$ cells $\left./ \mathrm{ml}\right)$ was seeded and grown on the coating surfaces. After being starved in media with $1 \%$ serum for $4 \mathrm{~h}$, confluent monolayers of HUVECs were wounded by scraping a pipet tip $(200 \mu \mathrm{L})$ across the monolayer to produce initial wounds with a constant diameter; they were then washed three times with PBS. The migration distance of the scratch was measured at $0,6,12$, and $24 \mathrm{~h}$ using ImageJ (NIH, US). The migration lengths were counted following the methods in our previous research ${ }^{38}$.

\section{Stent implantation in vivo}

Two groups of drug-coated stents were prepared using coaxial electrospraying: DPSs containing $50 \pm 2.36 \mu \mathrm{g}$ DTX (DPS-L) and DPSs containing $100 \pm 3.88 \mu \mathrm{g}$ DTX (DPS-H). The DTX content on the two stent groups was detected in the drug release after complete elution. Three control groups, BMS, BPS, and SES (DES with Sirolimus), were also prepared.

Twenty male Bama minipigs $(30-40 \mathrm{~kg}$ ) fed a normal diet were used in this study in accordance with the guidelines of the Chinese Animal Care and Use Committee standards. Three days before surgery, $150 \mathrm{mg}$ aspirin and $300 \mathrm{mg}$ clopidogrel were administered orally with feed. Before surgery, an intramuscular injection of xylazine hydrochloride $(0.15 \mathrm{~mL} / \mathrm{kg}, 30 \mathrm{mg} / 1.5 \mathrm{~mL})$ was given to each minipig for muscle relaxation. The venous channel was set up at the edge of the ear vein, and blood was taken simultaneously for a routine blood examination and serum biochemical examination, followed by ear intravenous administration of propofol at $8 \mathrm{ml} / \mathrm{h}$ for anesthesia. Minipigs were fixed to the operating table in a lateral position, and a $6 \mathrm{~F}$ introducer sheath was positioned in the right femoral artery under surgical exposure. Heparin sodium $(200 \mathrm{IU} / \mathrm{kg})$ was injected via the sheath. All catheters were subsequently introduced through the sheath and advanced to the left anterior descending (LAD)/left circumflex (LCX)/right coronary artery (RCA) through a guide wire. Baseline angiograms of each artery were obtained for each pig through quantitative coronary analysis (QCA). Prepared stents were deployed in the suitable vessel region during angiographic guidance. It was ensured that no branches were present in the stented region. Stents were dilated at $10 \mathrm{~atm}$. At the level of the balloon dilatation, the diameter of the aorta was approximately $2.5 \mathrm{~mm}$ and the expansion ratio of the aorta was 1:1.1. Two different stents were deployed per pig, and pre-deployment and post-deployment digital subtraction angiography was recorded. Gentamicin sulfate $(5 \mathrm{mg} / \mathrm{kg}$ ) was given as intramuscular injection in the next three days, and $150 \mathrm{mg}$ aspirin and $300 \mathrm{mg}$ clopidogrel were administered orally with feed every day. Animals were fed a normal diet after the intervention.

\section{Acquisition and observation of stent implantation samples}

Pigs were euthanized at 1,3 , and 6 months $(n=3$ at each time point for each stent group). At each time point, aortic specimens were excised transversely into two parts. One part was fixed in $4 \%$ paraformaldehyde and embedded in light-cured resin with hematoxylin and eosin stain (HE) for pathology organization analysis. Another part was fixed in $0.25 \%$ glutaraldehyde for $48 \mathrm{~h}$, and the morphology of the intima surface was observed by SEM. The proximal part and distal part of each stented vessel, myocardium, liver, spleen, lung, and kidney of pigs were fixed and sliced to check the safety of stents. Histomorphometric analysis was conducted on each section, including the vessel area $\left(\mathrm{mm}^{2}\right)$, internal elastic lamina (IEL) area $\left(\mathrm{mm}^{2}\right)$, lumen area $\left(\mathrm{mm}^{2}\right)$, neointima area $\left(\mathrm{mm}^{2}\right)$, and the neointima thickness $(\mathrm{mm})$ by Image Tool. The percentage of restenosis was calculated using the following equation:

$$
\text { Restenosis rate }(\%)=1-\frac{\text { lumen area }}{\text { internal elastic lamina area }} \times 100 \%
$$

Venous blood of pigs was collected for routine blood examination and serum biochemical examination.

\section{Statistical analysis}

At least three independent experiments were performed for the tests described above. Statistical analyses were conducted with GraphPad Prism 6. The differences 
between experimental groups were considered statistically significant when $p<0.05$. Unless indicated, the values are expressed as the mean $\pm \mathrm{SD}$.

\section{Results \\ Optimization of electrospraying parameters and preparation of dual-drug-loaded particles}

It was recognized that different parameters of the electrospraying process have a direct impact on the morphology of the particles. Because of the different viscosities of CS and PLGA, the formation of the Taylor cone at the coaxial nozzle was observed and recorded during spraying. The electrospraying parameters are shown in Table S1. Our results showed that microparticles were well separated at flow rates of $0.2 \mathrm{~mL} / \mathrm{h}$ (inner) and $0.6 \mathrm{~mL} / \mathrm{h}$ (outer), with a PLGA concentration of $10 \mathrm{mg} / \mathrm{mL}$, a CS concentration of $2.5 \mathrm{mg} / \mathrm{mL}$, a reception distance of $5 \mathrm{~cm}$ and a voltage of $14 \mathrm{kV}$ (Table S1) (Fig. 2a). The morphology of the hydrophobic core/hydrophilic shell particles was observed by SEM (Fig. 2b, c). The core/shell structure was viewed via confocal microscopy and TEM (Fig. $2 \mathrm{~d}-\mathrm{g}$ ). The particles were found to be well-dispersed, with most assuming uniform spheres with diameters of $643 \pm 34 \mathrm{~nm}$ and $1067 \pm 53 \mathrm{~nm}$ (Fig. 2h). The prepared particles dispersed evenly on the receiving surface with no large aggregation effect.

\section{Surface morphology of DPS}

The DPS had both green fluorescence (shell) and red fluorescence (core) distributed uniformly on the surface (Fig. 3a). The 316L SS stent surface was smooth and clean after pretreatment, and the surface was very uniform with no coating aggregation after coating (shown in Fig. 3b). Furthermore, after expansion, each coating was still homogeneous, smooth, and intact without cracking, tilting or shedding.

The change of surface morphology after each coating was investigated by AFM. After an acid pickling pretreatment, the $316 \mathrm{~L}$ SS stent surface was extremely uneven, with a surface roughness of $R a=30.6 \pm 0.26 \mathrm{~nm}$. However, the surface roughness significantly increased to $\mathrm{Ra}=43.2 \pm 0.42 \mathrm{~nm}$ after coating (Fig. 3c). Because the coating consisted of hydrophobic core/hydrophilic shell nano/micro particles, the surface became undulating and the contact angle of the $316 \mathrm{~L}$ SS surface increased from $39.65 \pm 2.19^{\circ}$ to $69.07 \pm 2.89^{\circ}$ (Fig. $3 \mathrm{~d}$ ).

Here, we mainly used FTIR to detect any functional group change of drugs and polymers before and after spraying that may have resulted from the high voltage and solvent changing the drug structure and inducing a chemical reaction between the drug and polymer. The main functional group of each of the drugs and polymers were clear: $-\mathrm{OH} /-\mathrm{NH}$ of $\mathrm{SZ}-21$ at $3410 \mathrm{~cm}^{-1},-\mathrm{OH} /-\mathrm{NH}$ superimposed absorption peak of chitosan at $3410 \mathrm{~cm}^{-1}$ and $\mathrm{CH}_{2}$ at $2925 \mathrm{~cm}^{-1}$ and $1467 \mathrm{~cm}^{-1}$ and $\mathrm{C}-\mathrm{O}$ at 1000 $\mathrm{cm}^{-1}$; additionally, several absorption peaks of DTX and PLGA were shown at $3400-3500 \mathrm{~cm}^{-1}$. SZ-21 and DTX mainly existed with polymers due to physical adsorption (Fig. 3e).

\section{Drug release from DPSs in vitro}

We tested the drug release characteristics of DPSs via a constant temperature horizontal rocking bed. Within the first $12 \mathrm{~h}$, the SZ-21 (shell) release was $26.70 \pm 0.61 \%$ of its initial loading, while the DTX (core) release was only $5.80 \pm 0.36 \%$ of its initial loading (Fig. 4a). The release of the shell drug dose rapidly reached a ratio of three times that from the core. By 7 day, the SZ-21 release had reached $37.9 \pm 0.74 \%$, while DTX release was still markedly slower at $17.70 \pm$ $0.73 \%$ (Fig. 4a). The release of two drugs was relatively stable for the next few days. After 28 days, the cumulative release of SZ-21 reached $62.30 \pm 0.58 \%$, and that of DTX reached $38.41 \pm 0.66 \%$. Throughout the 28 days, the cumulative release of SZ-21 was always higher than that of DTX.

To evaluate the drug release pattern of the coating, we carried out an analogue analysis. With the $M_{0}$ measured from our experiments, we conducted a standard least squares analysis, utilizing the analytical solution (1) in conjunction with the experimental data (Fig. 4b) to inversely estimate the unknown parameters $r, k_{1}$ and $k_{2}$ for which the model solution best fit the data for each drug. We observed that the release of each drug was well-described by this simple biphasic release model.

The surface topographies of DPSs were investigated by SEM after 0, 7, and 28 days (Fig. 4c). The stents presented a relatively smooth morphology prior to release ( 0 day). After 7 days, the morphology and glossiness of the stents showed no obvious differences with those from before, and the coating also had no obvious degradation. However, a shedding phenomenon appeared around the areas of light curvature ( 7 days). However, after 28 days, the surface of the coating displayed more particles, and the stent became uneven (yellow arrow and curve) because of the degradation of the coating ( 28 days).

\section{Blood compatibility of the DPC}

In general, platelet morphologies are related to their degree of activation. The number and morphology of platelets on the surface of DPC sheets were compared with $316 \mathrm{~L}$ SS and BPC (Fig. 5a, b). The DPC surface showed the least number of adherent platelets with a round shape and few pseudopodia. The DPC significantly decreased platelet activation, as seen through the expression of the platelet membrane glycoprotein CD62P (Fig. 5d). Further analysis of platelet type showed that 

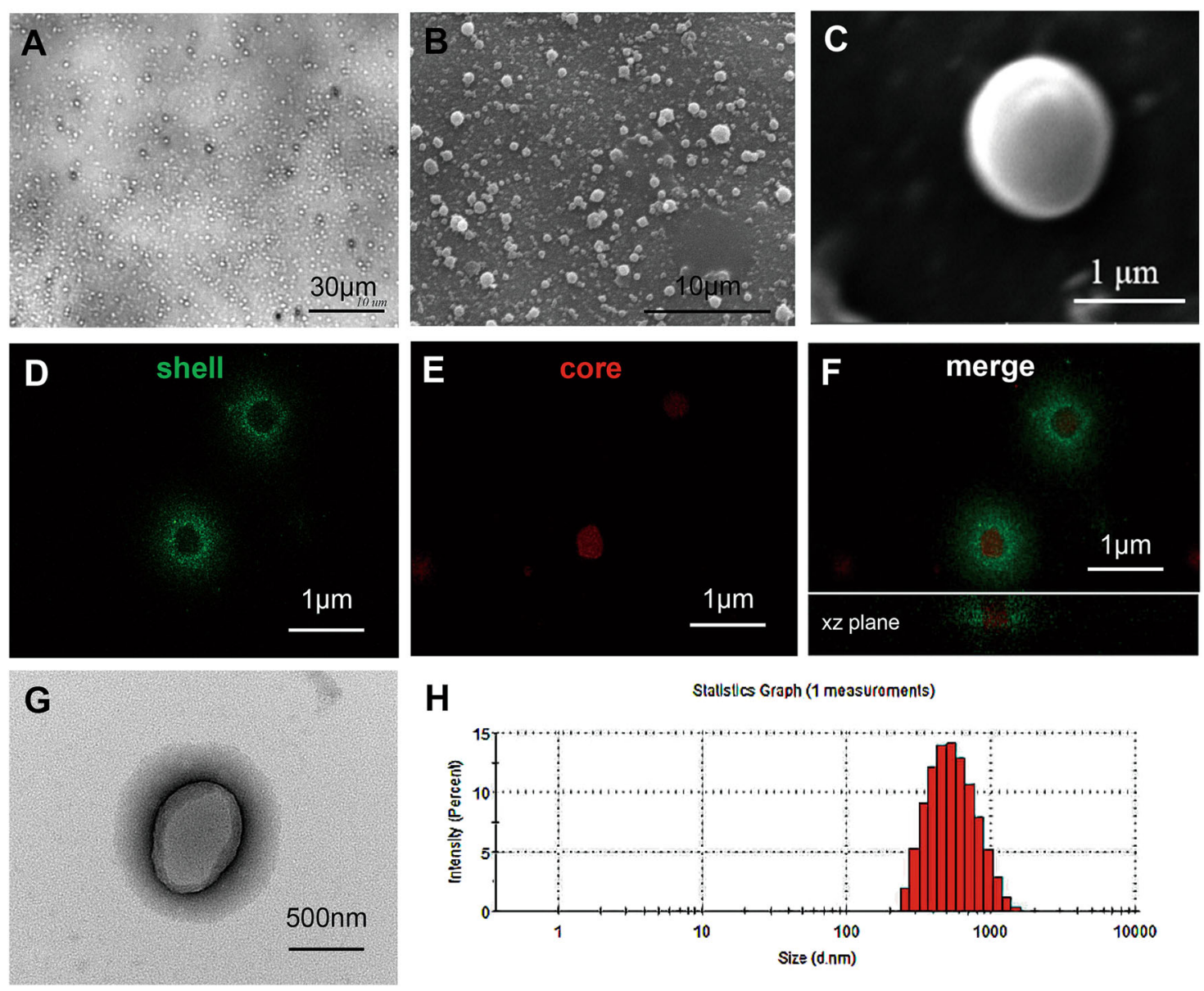

Fig. 2 The morphology and sizes of the SZ-21/DTX drug-loaded core/shell particles. The morphology of particles under the optical microscope (a) and SEM (b, c). $\mathbf{d}-\mathbf{g}$ The core/shell structure was shown with confocal microscopy $(\mathbf{d}-\mathbf{f})$ and TEM $(\mathbf{g})$.These particles were well separated and mostly spherical. The maximum diameter of these particles was nearly $1 \mu \mathrm{m}(\mathbf{h})$

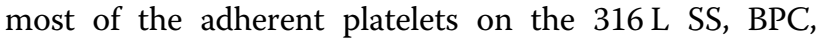
and DPC surfaces were types I-III (Fig. 5c), and the morphological indices of DPC $(2.83 \pm 0.26)$ were lower than those of $316 \mathrm{~L} \mathrm{SS}(2.93 \pm 0.18)$ and BPC $(2.91 \pm 0.21)$, which indicated less platelet activation on DPC. In addition, the hemolysis ratios of all samples were well within $1 \%$ (Fig. 5e), which suggested that DPC had excellent hemocompatibility. The APTT $(89.33 \pm 2.52 \mathrm{~s})$ of DPC were significantly prolonged compared with those of 316 L SS $(38.02 \pm 3.12 \mathrm{~s})$ and BPC $(52.33 \pm 3.05 \mathrm{~s})$. The PT of the three groups showed a similar trend of $26.67 \pm 1.61 \mathrm{~s}$ for DPC, $14.33 \pm 1.53 \mathrm{~s}$ for $316 \mathrm{~L}$ SS and $18.33 \pm 2.31 \mathrm{~s}$ for BPC (Fig. 5f, g).

\section{The proliferation and migration of HUVECs and HUASMCs on DPC}

The structure of the hydrophobic core/hydrophilic shell particles ensured that the anti-proliferative drug in the core released very slowly, having little influence on
HUVEC adhesion and proliferation. In fact, the coating showed a promotion of HUVEC proliferation in the early days (Fig. 6a), while the low dose of DTX significantly inhibited the proliferation of HUVSMCs (Fig. 6b). Figure S1 (A-F) showed an SEM image of HUVEC adhesion on the BMSs, BPSs, and DPSs. After 5 days, cells were spindle-shaped or irregular triangleshaped on the surface of the three groups, and the number of cells had no significant difference. The NO release and T-AOC of HUVECs were not influenced by the release of DTX (Figure S1G, H).

The migration ability of HUVECs and HUASMCs on $316 \mathrm{~L} \mathrm{SS}, \mathrm{BPC}$, and DPC were tested in a scratch wound model in vitro. Due to the release of DTX, the migration distances of HUVECs in the DPC and BPC groups were less than those of the control group (Fig. 6c, e). The inhibition of HUASMC growth in the DPC group was obvious, the intercellular space was increased and the number of cells was less than those in the other two 

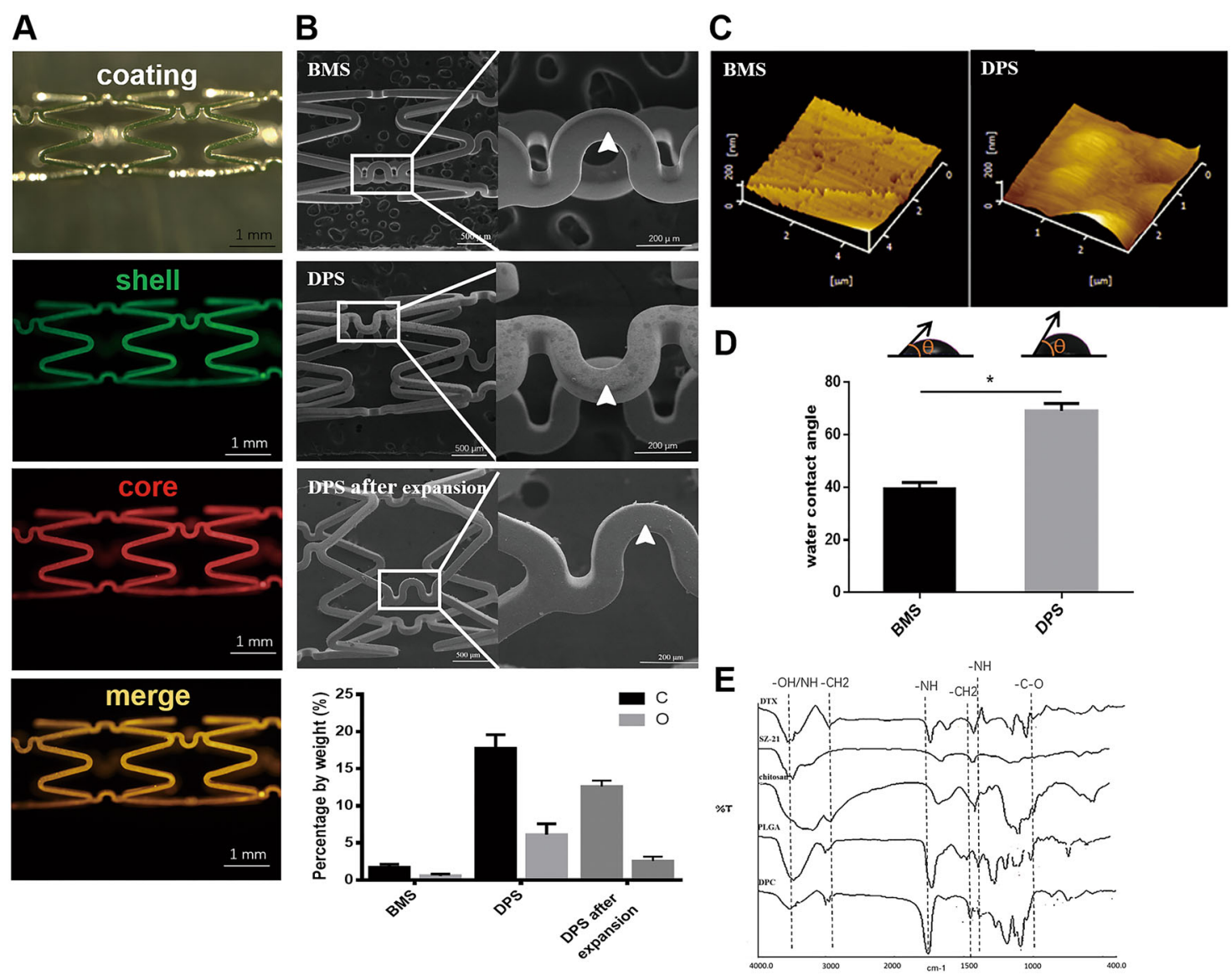

Fig. 3 Evaluation of the surfaces of different stent coatings. a Core (red)/shell (green) drug-loaded particles were prepared on the surface of stents by coaxial electrospraying. $\mathbf{b}$ The surface of BMSs was smooth and uniform, while the DPS surface was roughened and exhibited no loss, tilt and cracking in the binding parts of different stents after the expansion. The carbon and oxygen weight percentages of DPS before and after expansion are shown in the image. c The roughness of DPSS (right) increased compared to that of the $316 \mathrm{~L} \mathrm{SS}$ (left) observed by atomic force microscopy. d The contact angle of DPSs significantly increased but was still less than $90^{\circ}$, which means that it remained hydrophilic. e FTIR of drug coating for the DTX, SZ-21, chitosan PLGA, and particle coating. The main functional group of each of the drugs and polymers existed; SZ-21 and DTX mainly existed with polymers in the form of physical adsorption

groups. During the 24-h migration, the migration length of HUASMCs in the DPC group was inhibited and notably delayed compared with the other two groups (Fig. 6d, f).

\section{In vivo stent implantation and animal survival after surgery}

In total, 36 stents (number of stents in each group >5) were implanted successfully into 20 Bama minipigs (Figure S2). Subsequently, the animals were revived and returned to the breeding center for normal feeding. During the feeding period, all physiological indices of porcine health, such as diet and weight, were normal. There were at least two different stents implanted in the coronary of each minipig; the main indicators of routine blood examination and serum chemical examination exhibited no significant changes after stent implantation for 12 months. The color, luster, and hardness of heart, liver, spleen, lung, and kidney were normal, and the HE stain indicated no obvious organic issues. These data showed that all stents were safe and had no significant toxicity (Table S2, S3, Figure S3).

\section{Coronary angiography}

Coronary angiography was performed after stent implantation immediately and at 1,3, and 6 months. After stent implantation for 1, 3, and 6 months, angiography showed that all of the vascular walls were smooth, with no obverse in-stent thrombosis, and there were no signs of stent malapposition in any of the groups (stent is shown with blue arrow) (Fig. 7a). The rate of lumen loss after 1 month showed no significant difference among all groups $(p<0.05)$ (Fig. 7b). After 3 months, the BPSs showed a significant difference from the DPS-H group 


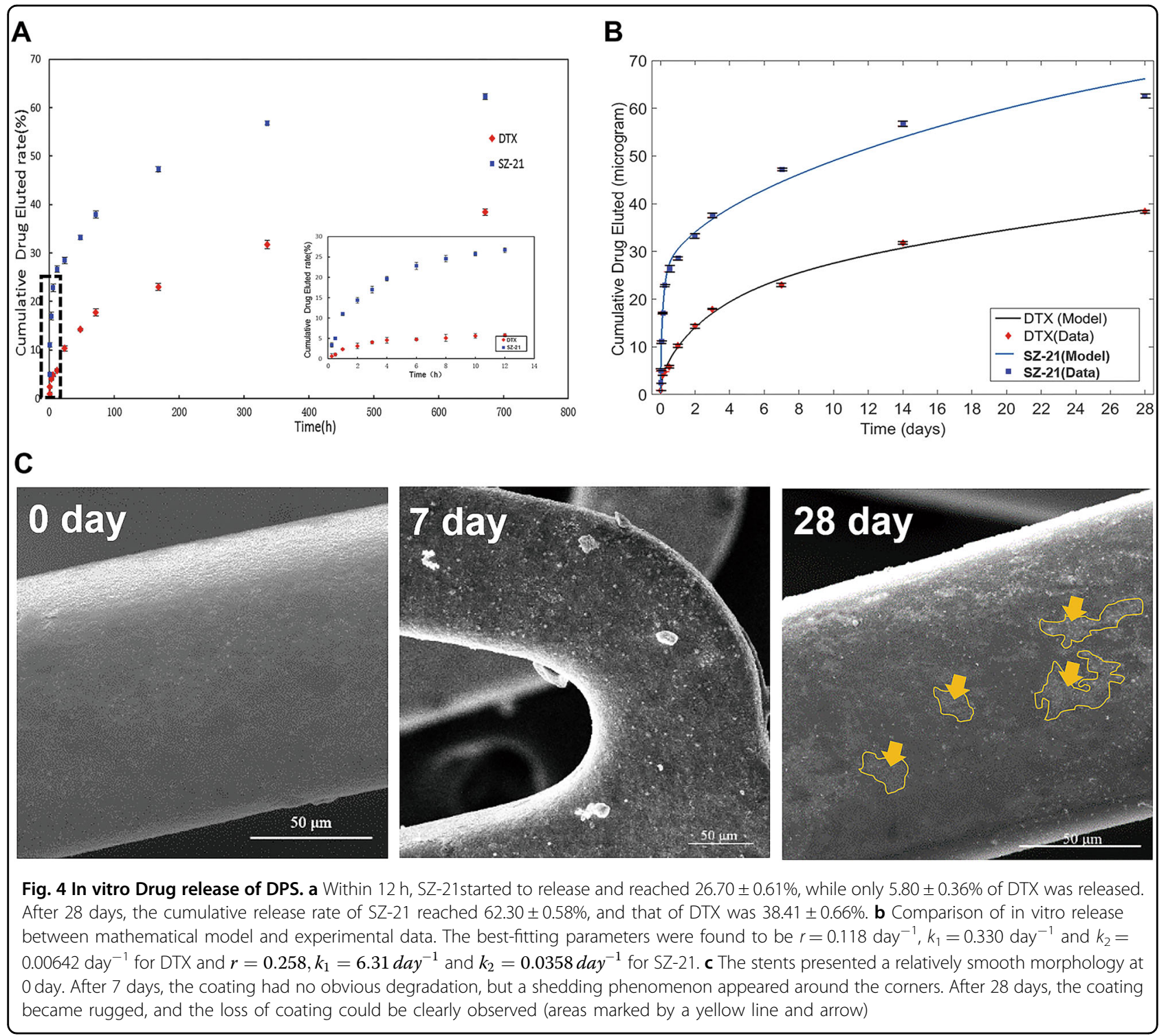

$(p<0.05)$ (Fig. 7c). The rate of lumen loss after 6 months showed that the BPS and SES groups exhibited a significant difference compared with the DPS-H group $(p<0.05)$ (Fig. 7d).

\section{DPS promoted re-endothelialization in vivo}

After implantation for $1,3,6$, and 12 months, the progress of re-endothelialization was examined by SEM. The progress of re -endothelialization of coronary in all groups was almost completed within 1 month, and the surfaces of all stent groups were covered partially with cobblestonelike endothelial cells. After 3 and 6 months, the DPSs-H had adhered to them a small amount of platelet and fibrin, and the morphology of the adhered cells became oval following the initial roundness. The BPSs and SESs seemed to have clearer stent shapes (Fig. 8 and Figure S4).
These data suggested that the antiplatelet aggregation and re-endothelialization were enhanced in animals treated with DPS and not adversely affected by the loaded DTX. After implantation for 12 months, the surfaces of the BMS, BPS, and DPS-H groups were more or less covered with some platelet and fibrin, while the surface on the BMS group had the maximum amount of platelet and fibrin. Most cells on the surface of the BMS were spindle-shaped, which was different from the other groups (Figure S5).

\section{Reduced in-stent restenosis in porcine coronary arteries after DPS implantation}

To evaluate the effects of DTX eluted from stents in vivo, a histomorphometric analysis was performed in the BMS, BPS, SES, and DPS group after implantation for 


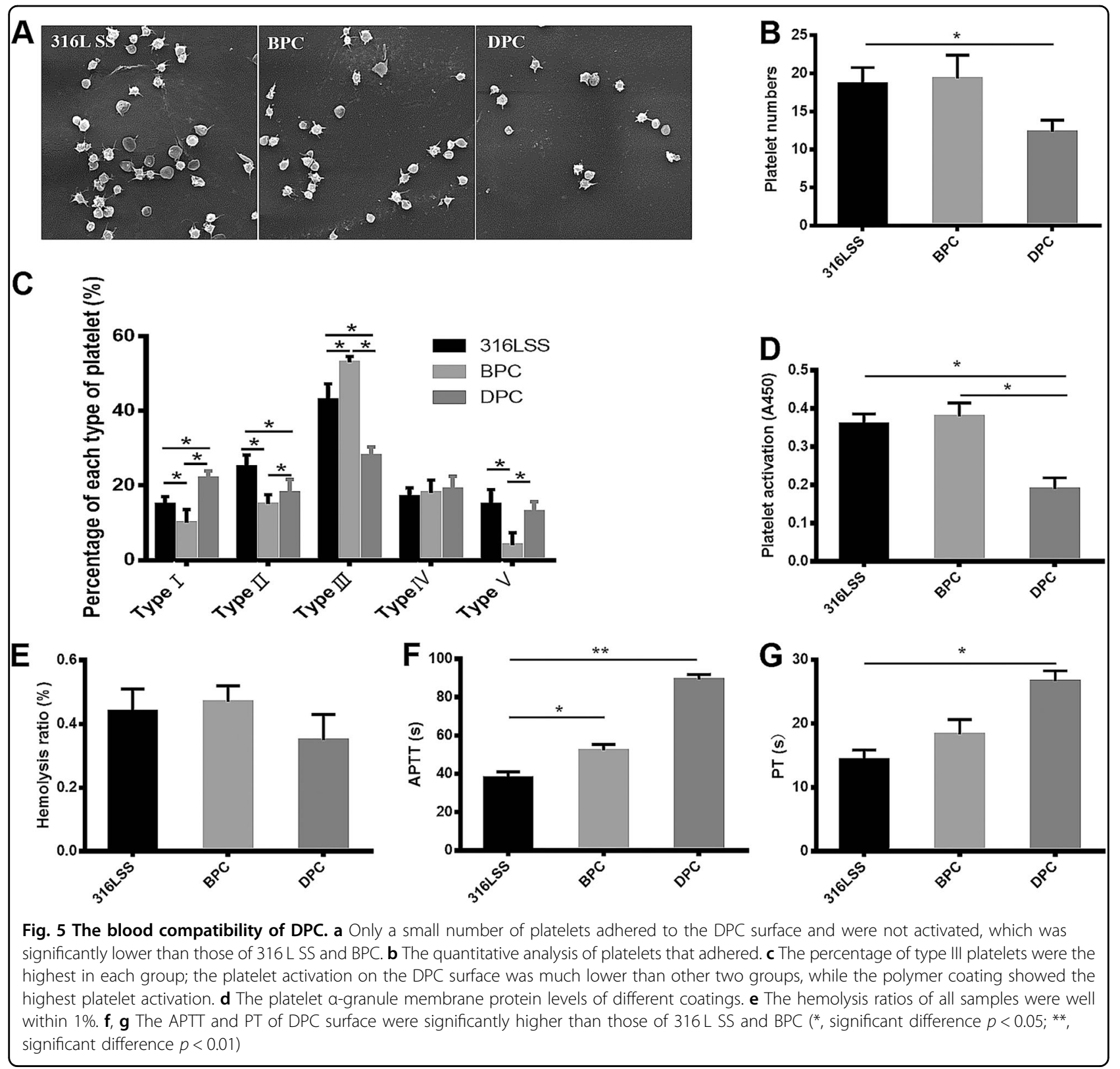

1 and 3 months (Fig. 9a). Endothelial repair was completed in all groups, and the stent surface was covered with intact intima. Compared to that of BMS and BPS, the elution of DTX from DPS-Hs significantly reduced the mean neointimal area and the percentage luminal stenosis and showed a similar anti-restenosis effect (Fig. 9b, c). Above all, the DPS-Hs had a better anti-restenosis effect, while the BPSs produced the poorest results. After implantation for 6 months, there was no thrombosis or obvious intimal hyperplasia in the groups. No statistically significant difference was observed between the BMS and DPS groups (Figure S6).

\section{Discussion}

The introduction of DESs led to a significant reduction in restenosis and demonstrated early improvements in safety after $\mathrm{PCI}^{39}$. However, their use is associated with an increased risk of late ST and considerable restenosis rates ${ }^{40}$; these remaining issues have driven the design of new stent technology ${ }^{41}$. In the present report, we described a novel DES coating consisting of particles with a core of the anti-proliferative drug DTX and a shell of SZ-21 deposited by coaxial electrospraying, which provides the biphasic diffusion-controlled release of each drug to effectively reduce in-stent restenosis and thrombosis. 


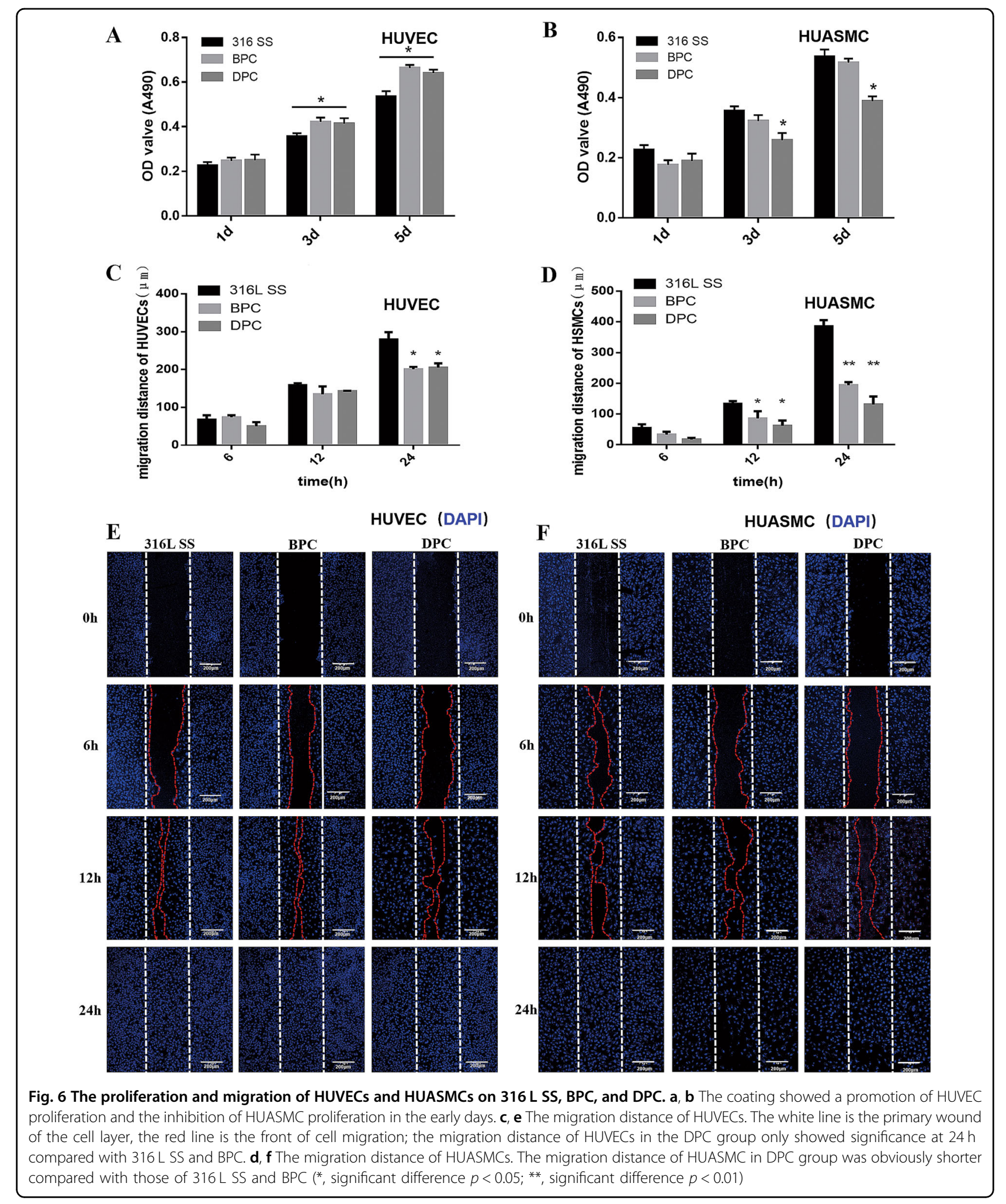

After PCI, the injured vessel undergoes a variable combination of platelet activation and acute thrombosis, intimal proliferation, vascular remodeling/wound contraction, and recoil of the normal section of the $\operatorname{artery}^{42}$. For the second generation of DESs, whose coatings mostly delivered paclitaxel and sirolimus 


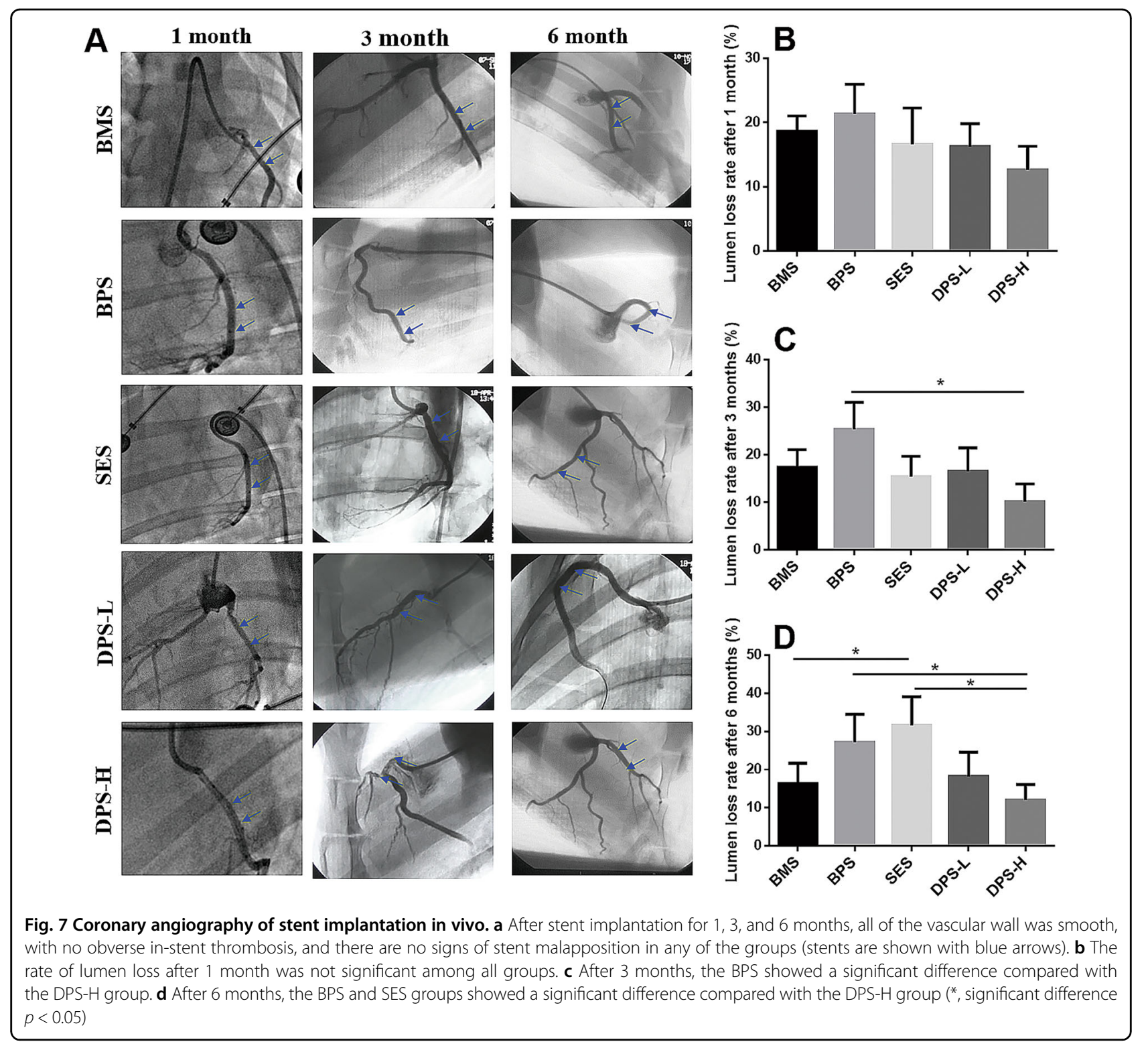

together with their analogues ${ }^{43}$, the coating could only achieve short-term anti-proliferative function with delayed re-endothelialization. Most vascular stent coatings that have been produced have been given only one kind of function, such as the inhibition of inflamma$\operatorname{tion}^{44,45}$, promotion of re-endothelialization ${ }^{46}$ or a novel way of preventing in-stent restenosis ${ }^{47}$. In our design, we arranged the coating to have dual anti-thrombosis and anti-restenosis functions. The efficient platelet GP IIb/IIIa receptor monoclonal antibody SZ- $21^{36}$ and antiproliferative paclitaxel analogues $\mathrm{DTX}^{48,49}$ were chosen for anti-thrombosis and anti-restenosis, respectively. To prepare core/shell structure particles for both hydrophilic and hydrophobic drugs, we chose CS as the carrier of SZ21 to be the shell and PLGA as the carrier of DTX to be the core. The CS is an appropriate carrier for protein and other activated materials ${ }^{50,51}$, and PLGA is an FDAapproved carrier.

The most popular preparation methods for stent coating include dipping ${ }^{52}$ and spraying ${ }^{53,54}$, but such methods are unwieldy considering the hydrophobicity and hydrophilicity of the drugs. Hossfeld et al. ${ }^{55}$ prepared a bioactive coronary stent coating based on layer-by-layer technology for siRNA release, whereas Song et al. ${ }^{56}$ demonstrated multi-step immersion for the simultaneous coating of VEGF and anti-CD34 antibody. In this study, we sought to fabricate a novel hydrophobic core/hydrophilic shell particle coating on the surface of the implant using coaxial electrospraying. Moreover, the core/shell is interchangeable according to the research purpose and 


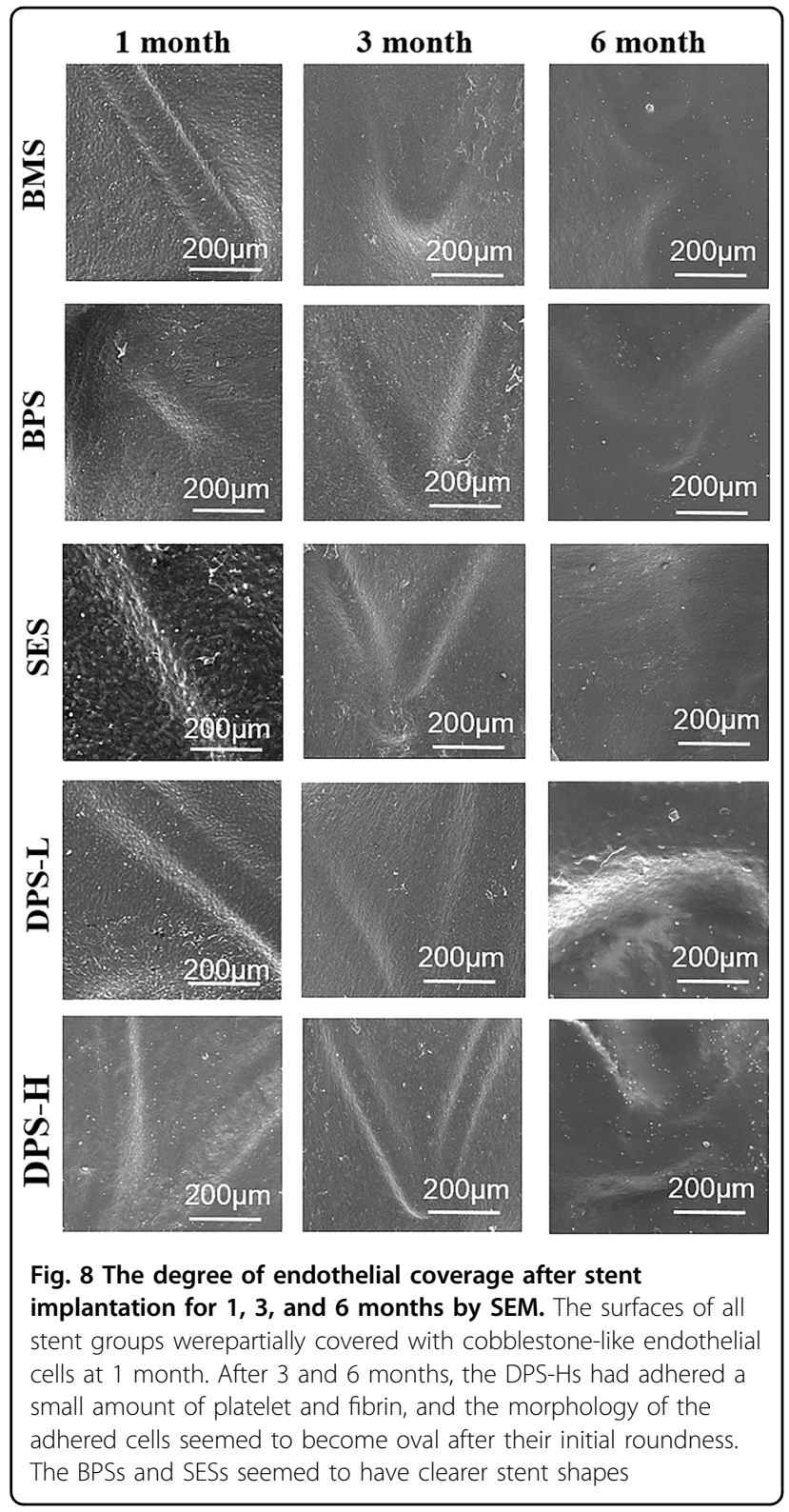

the design of experiments by altering the parameters of coaxial electrospraying ${ }^{57}$. We focused on the type and concentration of polymer, voltage, flow rate, and reception distance; these factors control the coating and ensure precision as well as reproducibility ${ }^{58}$. The voltage affected the formation and stabilization of the Taylor cone. The feeding speed of the sample influenced the formation and size of the particles, directly affecting the quality of the coating. Particles obtained via electrospraying were well separated and most spherical.

The surface of the stent contacts the blood and vascular tissue synchronously, and it is known that the coating influences the long-term outcomes of stent implantation $^{59}$. We observed the distribution of green fluorescence and red fluorescence on the stent. The results demonstrated that the coating was excellent, with a uniform surface. Changes in surface hydrophilicity may cause quantitative and qualitative variations in absorbed proteins, which can directly influence the biocompatibility of the materials ${ }^{60}$. To evaluate the changes in hydrophilicity of the surface after each coating, the water contact angles on material surfaces were measured. After coating, while CS was a hydrophilic material and contributed to the shell particles, the contact angles of the coating were less than $90^{\circ}$; the coating therefore retained its hydrophilicity. The aggregation and activation of adherent platelets are considered key indicators for evaluating the hemocompatibility of a material. Our results indicated that the SZ-21 on DPSs could inhibit platelet adhesion and aggregation and prolong APTT, PT, and TT values, which suggested that SZ-21 may contribute to inhibiting the thrombosis of the stent section after DPS implantation.

Nano/micro particles have previously been used to achieve multi-drug delivery from drug delivery systems ${ }^{61}$. In this study, we loaded two drugs with hydrophilic differences via coaxial electrospraying. The solvent of PLGA and DTX in the core of particles was dichloromethane; the solvent of CS and drug SZ-21 in the shell was acetic acid. The release profiles of the drugs were found to be markedly different, with SZ-21 being released faster, followed by DTX. The hydrophobic core/hydrophilic shell particles controlled the release of two drugs to realize a differential release, which may well hold advantages for adequate vessel repair over a single drug.

We have shown that the in vitro DPS was a diffusioncontrolled release system well-described by a biphasic mathematical model. Moreover, we have inversely estimated the parameters associated with the model. The model suggested that for both drugs, the majority of the drug was released initially within the slow-release phase (88.2\% for DTX and $74.2 \%$ for SZ-21). In the case of DTX, the fast phase release rate was more than 50 times greater than the slow release rate $\left(k_{1}=0.330\right.$ day $^{-1}, k_{2}=$ $0.00642 \mathrm{day}^{-1}$ ) while for SZ-21, the corresponding ratio was more than $176\left(k_{1}=6.31\right.$ day $^{-1}, k_{2}=0.0358$ day $\left.^{-1}\right)$. Comparing the two drugs, the fast phase release rate of SZ-21 was more than 19 times greater than that of DTX, while the slow release rate was over five-fold greater for SZ-21. While such modeling allows for the timedependent prediction of drug release for core/shell coated stents prepared in the same way as this study, it did not allow one to predict how changes to the core/shell surface preparation would influence the release. For example, if as a result of variations in the surface preparation methods we obtain different particle sizes, then we are not able to use this model to predict the effect on the release profile, since our mathematical solution is in terms of bulk (or 'effective') parameters. Nevertheless, we anticipate that 

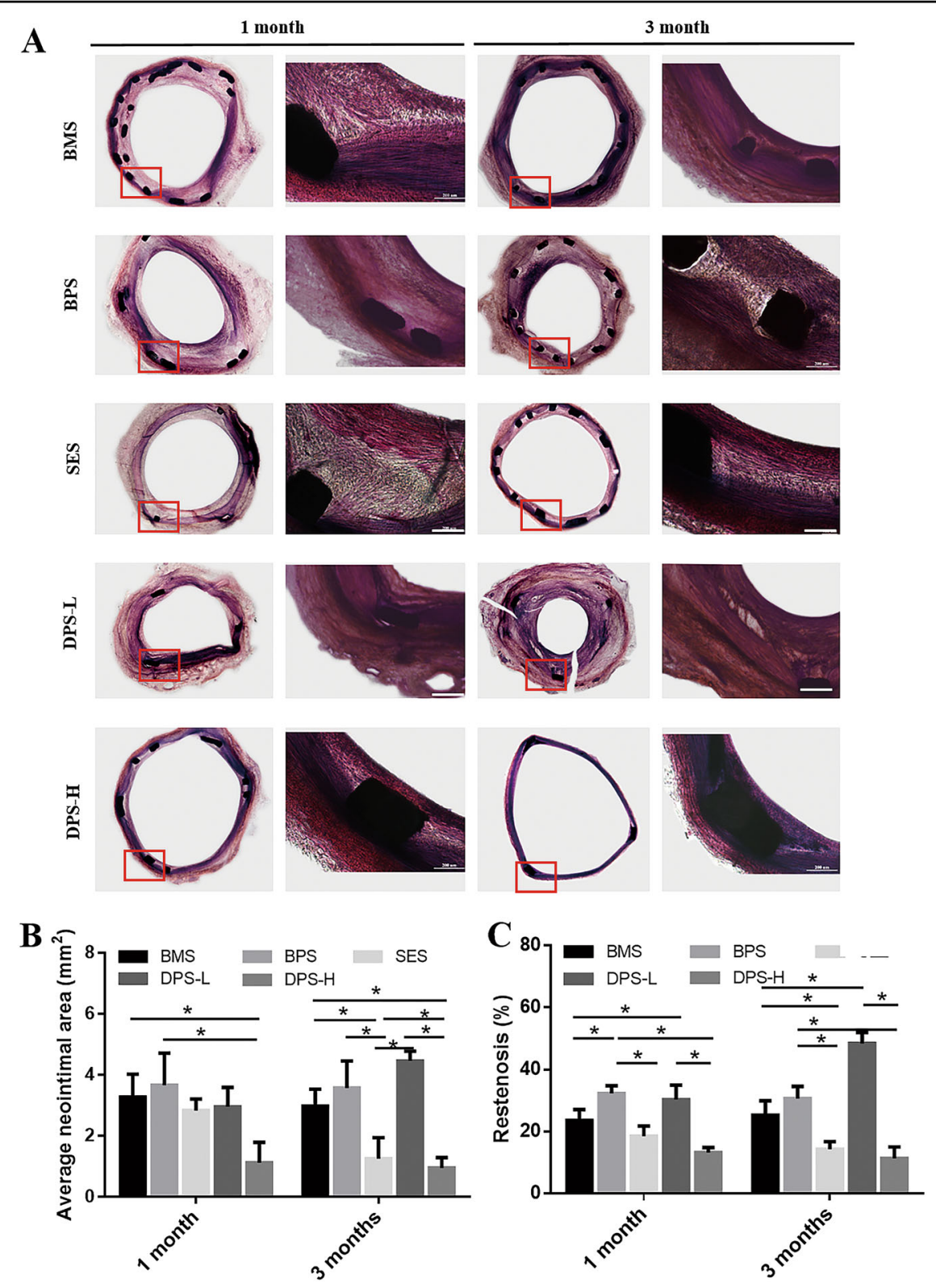

Fig. 9 Histomorphometric analysis results of BMS, BPS, SES, and DPS after stent implantation for 1 and 3 months. a DPS-Hs inhibited intimal hyperplasia; the orange triangle turned to inflammatory cells around the stent strut. $\mathbf{b}$ The neointimal area of DPS was significantly less than those of the other groups. $\mathbf{c}$ The stenosis rate of DPS was significantly less than those in the other groups. This indicated that the DPS-H inhibited neointimal hyperplasia and restenosis ( $\mathrm{S}$ stent, $\mathrm{N}$ neointima, L lumen, $\mathrm{M}$ media) $\left(^{*}\right.$, significant difference $p<0.05$ )

this model will still be very useful in these cases. For example, if the parameters of the coating preparation process are modified, then we may obtain experimental data at a smaller number of time points and extrapolate them to later time points. Furthermore, a validated model of drug release in vitro may be extrapolated to in vivo by coupling the drug release model to a model of drug uptake in arterial tissue (see for example ${ }^{62}$ ).

In the early period after stent implantation, platelets are activated and tend to adhere to the injured intima. A large release of SZ-21 within the first $12 \mathrm{~h}$ was achieved for the inhibition of platelets to avoid thrombosis. Simultaneously, as the endothelial cells begin to adhere to the surface of the DPS, the modest release of DTX did not influence the adhesion. Along with the increased release of DTX during the following period, the proliferation and migration of HUASMCs were inhibited from adhering to the intima to avoid excessive endometrial hyperplasia. The differential release of the two drugs was consistent with the results in vivo. As approximately $62 \%$ of 
SZ-21was released in vitro over 28 days, the least number of adherent platelets and fibrinogen on DPS was observed in 1 month accordingly, we could infer a similar drug release trend in vivo.

The ideal drug release mode should conform to the healing progress of the damaged endothelium, avoiding the risk of stent restenosis and late thrombosis caused by prolonged vascular inflammation and the VSMC migration into the intima. Good drug loading and delivery methods are beneficial to accelerate endothelial repair. The combined use of antithrombotic and antiproliferative drugs on stents achieved better repair after implantation. In our in vivo study, using a porcine model for the better simulation of coronary stent implantation, these demands of DPSs were evaluated. The angiography showed no obvious in-stent thrombosis in the DPS group during the entire observation stage $(1,3$, and 6 months) compared with the BMSs and traditional DESs in other studies $^{63}$. When seen from the surface of the neointima in the DPC group (1, 3, 6 months), there was less adhesion of platelet and fibrinogen. Despite the dual antiplatelet therapy, our DPS loaded with SZ-21indicated the better effect of anti-coagulation and thrombolytic therapy for early and late thrombosis than traditional DESs and may have similar applications as other anti-thrombotic coating stents (such as heparin-loaded) ${ }^{64}$. Furthermore, the DPS also reduced the risk of stent restenosis. There was a reduced lumen loss rate in the DPS- $\mathrm{H}$ group compared with the other groups from 1 to 6 months. The neointimal area of the DPS-Hs of DTX was significantly reduced compared with those of BMSs and BPSs. The two doses of DTX allowed for the evaluation of the relationship between drug doses, drug release and antiproliferative effect. The DPS-H group showed fast reendothelialization with the cobblestone-like endothelial cell layer during the first month, while the BMS and BPS groups showed incomplete re-endothelialization in some local regions. The high dose of DTX not only showed no prevention of re-endothelialization but also effectively inhibited intimal hyperplasia because of the diffusioncontrolled release system. This drug release system for implant coating may therefore reduce or avoid the side effects induced by a high dose of anti-proliferative drugs in conventional coatings ${ }^{65}$. The dual-functional DPSs with high drug dose showed better application potential compared with BMSs and SESs in vivo.

\section{Conclusion}

Hydrophobic core/hydrophilic shell SZ-21/DTX dualdrug-loaded stents exhibiting a uniform coating of nano/ micro particles using coaxial electrospraying were prepared. After balloon dilatation, the coating remained smooth and uniform. The majority of the drugs were initially released within the slow-release phase; the fast phase release rate of SZ-21 was greater than that of DTX, while the slow release rate was over five-fold greater for SZ-21. The differences between the fast and slow release rates for SZ-21 and DTX clearly highlight the biphasic nature of the release. The DPSs showed good blood and cell compatibility, promoted re-endothelialization in vivo and reduced in-stent restenosis in porcine coronary arteries. Coaxial electrospraying is a one-step and effective technique for stent coating with multiple drugs and has great potential to create the enhanced implants of the future.

\section{Acknowledgements \\ This study is supported by grants from the National Key Research and Development Program of China (2016YFC1102305), the National Natural Science Foundation of China (11332003), the Visiting Scholar Foundation of the Key Laboratory of Biorheological Science and Technology, Ministry of Education (CQKLBST-2016-003), the Fundamental Research Funds for the Central Universities (106112017CDJPT230001, 106112017CDJZRPY0202), and the National Key Technology R\&D Program of China (2012BAl18B02) as well as the Science and Technology Commission of Yuzhong District in Chongqing (20150135). We highly appreciate the kind assistance from Professor Changgeng Ruan at the Jiangsu Institute of Hematology, Professor RS Nowakowski at Florida State University and Professor Hossam Haick at Technion-Israel Institute of Technology. We are also thankful for the support from the Chongqing Engineering Laboratory in Vascular Implants and the Public Experiment Center of State Bioindustrial Base (Chongqing).}

\begin{abstract}
Author details
${ }^{1}$ Key Laboratory for Biorheological Science and Technology of Ministry of Education, State and Local Joint Engineering Laboratory for Vascular Implants, Bioengineering College of Chongqing University, Chongqing 400030, China. ${ }^{2}$ The Affiliated Central Hospital of Chongqing University and Chongqing Emergency Medical Center, Chongqing 400014, China. ${ }^{3}$ Center of Cardiology, Chongqing People's Hospital, Chongqing 400013, China. ${ }^{4}$ Division of Biomedical Engineering, University of Glasgow, Glasgow, UK. ${ }^{5}$ Istituto per le Applicazioni del Calcolo - CNR, Via dei Taurini 19, 00185 Roma, Italy
\end{abstract}

Conflict of interest

The authors declare that they have no conflict of interest.

Publisher's note

Springer Nature remains neutral with regard to jurisdictional claims in published maps and institutional affiliations.

Supplementary information is available for this paper at https://doi.org/ 10.1038/s41427-018-0064-z.

Received: 22 February 2018 Revised: 22 May 2018 Accepted: 28 May 2018. Published online: 19 July 2018

\section{References}

1. O'brien, B. \& Carroll, W. The evolution of cardiovascular stent materials and surfaces in response to clinical drivers: a review. Acta Biomater. 5, 945-958 (2009).

2. Bønaa, K. H. et al. Drug-eluting or bare-metal stents for coronary artery disease. New Engl. J. Med. 375, 1242-1252 (2016).

3. Douglas, P. S. et al. Clinical effectiveness of coronary stents in elderly persons: results from 262,700 Medicare patients in the American College of CardiologyNational Cardiovascular Data Registry. J. Am. Coll. Cardiol. 53, 1629-1641 (2009).

4. Erruys, P. W. et al. 5-year clinical outcomes of the ARTS II (Arterial Revascularization Therapies Study II) of the sirolimus-eluting stent in the treatment of 
patients with multivessel de novo coronary artery lesions. J. Am. Coll. Cardiol. 55, 1093-1101 (2010).

5. Stettler, C. et al. Outcomes associated with drug-eluting and bare-metal stents: a collaborative network meta-analysis. Lancet 370, 937-948 (2007).

6. Camenzind, E., Steg, P. G. \& Wijns, W. Stent thrombosis late after implantation of first-generation drug-eluting stents: a cause for concern. Circulation 115 1440-1455 (2007)

7. Baber, U. et al. Coronary thrombosis and major bleeding after $\mathrm{PCl}$ with drugeluting stents: risk scores from PARIS. J. Am. Coll. Cardiol. 67, 2224-2234 (2016).

8. Inoue, T. et al. Comparison of activation process of platelets and neutrophils after coronary stent implantation versus balloon angioplasty for stable angina pectoris. Am. J. Cardiol. 86, 1057-1062 (2000).

9. Park, E. S. et al. Inhibitory effects of docetaxel on platelet-derived growth factor (PDGF)-BB-induced proliferation of vascular smooth muscle cells through blocking PDGF-receptor $\beta$ phosphorylation. J. Pharmacol. Sci. 116, 204-213 (2011).

10. Yasuda, S. et al. Local delivery of low-dose docetaxel, a novel microtubule polymerizing agent, reduces neointimal hyperplasia in a balloon-injured rabbit iliac artery model. Cardiovasc Res. 53, 481-486 (2002).

11. Skinner, M. P. Thrombosis and thrombolysis: platelet membrane glycoproteins. Heart Lung Circ. 16, 176-179 (2007).

12. Aggarwal, R. K. et al. Antithrombotic potential of polymer-coated stents eluting platelet glycoprotein Ilb/llla receptor antibody. Circulation 94, 3311-3317 (1996).

13. Boersma, E. et al. Platelet glycoprotein Ilb/lla inhibitors in acute coronary syndromes: a meta-analysis of all major randomised clinical trials. Lancet $\mathbf{3 6 0}$, 342-343 (2002).

14. Lim, K. S. et al. Effect of polymer-free $\mathrm{TiO}_{2}$ stent coated with abciximab or alpha lipoic acid in porcine coronary restenosis model. J. Cardiol. 64, 409-418 (2014).

15. Freiberg, S. \& Zhu, X.X. Polymer microspheres for controlled drug release. Int J. Pharm. 282, 1-18 (2004).

16. Arsiwala, A., Desai, P. \& Patravale, V. Recent advances in micro/nanoscale biomedical implants. J. Control Release 189, 25-45 (2014).

17. Natarajan, J. V., Nugraha, C., Xu, W. N. \& Venkatraman, S. Sustained-release from nanocarriers: a review. J. Control Release 193, 122-138 (2014).

18. Yang, J. et al. The prevention of restenosis in vivo with a VEGF gene and paclitaxel co-eluting stent. Biomaterials 34, 1635-1643 (2013).

19. Kanzer, J. et al. In situ formation of nanoparticles upon dispersion of melt extrudate formulations in aqueous medium assessed by asymmetrical flow field-flow fractionation. J. Pharm. Biomed. 53, 359-365 (2010).

20. Zhang, J., LV, H., Jiang, K. \& Gao, Y. Enhanced bioavailability after oral and pulmonary administration of baicalein nanocrystal. Int J. Pharm. 420, 180-188 (2011).

21. Müller, R. H., Mäder, K. \& Gohla, S. Solid lipid nanoparticles (SLN) for controlled drug delivery - a review of the state of the art. Eur. J. Pharm. Biopharm. 45, 149-155 (1998).

22. Danhier, F. et al. Paclitaxel-loaded PEGylated PLGA-based nanoparticles: in vitro and in vivo evaluation. J. Control Release 133, 11-17 (2009).

23. Thian, E. S. et al. Electrospray deposition of nanohydroxyapatite coatings: a strategy to mimic bone apatite mineral. Thin Solid Films. 519, 2328-2331 (2011).

24. Almería, B., Fahmy, T. M. \& Gomez, A. A multiplexed electrospray process for single-step synthesis of stabilized polymer particles for drug delivery. J. Control Release 154, 203-210 (2011).

25. Dong, M. K. et al. Fabrication and controlled release of electrosprayed ReoProloaded metal surface for vascular stent. Macromol. Res. 19, 501-506 (2011).

26. Zamani, M., Prabhakaran, M. P., Varshosaz, J., Mhaisalkar, P. S. \& Ramakrishna, S. Electrosprayed Montelukast/poly (lactic-co-glycolic acid) particle based coating: a new therapeutic approach towards the prevention of in-stent restenosis. Acta Biomater. 42, 316-328 (2016).

27. Sun, D. et al. Coronary drug-eluting stents: from design optimization to newer strategies. J. Biomed. Mater. Res A. 102, 1625-1640 (2014).

28. Tan, A., Alavijeh, M. S. \& Seifalian, A. M. Next generation stent coatings: convergence of biotechnology and nanotechnology. Trends Biotechnol. 30 406-409 (2012).

29. Ding N. US patent 5980972 (1999).

30. Meng, S. et al. The effect of a layer-by-layer chitosan-heparin coating on the re-endothelialization and coagulation properties of a coronary stent system. Biomaterials 30, 2276-2283 (2009).
31. Cao, Y., Wang, B. C., Wang, Y. Z. \& Lou, D. S. Polymer-controlled core-shell nanoparticles: anovel strategy for sequential drug release. RSC Adv. 4, 30430-30439 (2014)

32. An, G. Y., Dong, N. Z., Shao, B. J., Zhu, M. Q. \& Ruan, C. G. Expression and characterization of the SCFv fragment of antiplatelet GPIIla monoclonal antibody SZ-21. Thromb. Res. 105, 331-337 (2002).

33. Xi, X. et al. Competitive binding of a monoclonal antibody SZ-21 with antiPLA1 antibodies and its potential for clinical application. Nouv. Rev. Fr. Hematol. 34, 239-242 (1992).

34. Saylor, D. M., Adidharma, L., Fisher, J. W. \& Brown, R. P. A biokinetic model for nickel released from cardiovascular devices. Regul. Toxicol. Pharm. 80, 1-8 (2016).

35. Wang, H. G. et al. Biofunctionalization of titanium surface with multilayer films modified by heparin-VEGF-fibronectin complex to improve endothelial cell proliferation and blood compatibility. J. Biomed. Mater. Res A. 101, 413-420 (2013).

36. Gawaz, M. et al. Changes in membrane glycoproteins of circulating platelets after coronary stent implantation. Heart 76, 166-172 (1996).

37. Ko, T. M., Lin, J. C. \& Cooper, S. L. Surface characterization and platelet adhesion studies of plasma-sulphonated polyethylene. Biomaterials 14, 657-664 (1993).

38. Wu, X. et al. Re-Re-endothelialization Study on Endovascular Stents Seeded by Endothelial Cells through Up- or Downregulation of VEGF. ACS Appl. Mater. Interfaces 8, 7578-7589 (2016).

39. Garg, S. \& Serruys, P. W. Coronary stents: current status. J. Am. Coll. Cardiol. $\mathbf{5 6}$ S1-S42 (2010).

40. Lee, J. M. et al. Comparison among drug-eluting balloon, drug-eluting stent, and plain balloon angioplasty for the treatment of in-stent restenosis: a network meta-analysis of 11 randomized, controlled trials. JACC-Cardiovasc Inte $\mathbf{8}$, 382-394 (2015)

41. Puricel, $\mathrm{S}$. et al. Comparison of everolimus- and biolimus-eluting coronary stents witheverolimus-eluting bioresorbable vascular scaffolds. J. Am. Coll. Cardiol. 65, 791-801 (2015).

42. Wilensky, R. L. et al. Vascular injury, repair, and restenosis after percutaneous transluminal angioplasty in the atherosclerotic rabbit. Circulation $\mathbf{9 2}$, 2995-3005 (1995)

43. Wykrzykowska, J. J., Onuma, Y. \& Serruys, P. W. Advances in stent drug delivery: the future is in bioabsorbable stents. Expert Opin. Drug Del. 6, 113-126 (2009).

44. Ali, M. T. et al. A novel CX3CR1 antagonist eluting stent reduces stenosis by targeting inflammation. Biomaterials 69, 22-29 (2015).

45. Slee, J. B. et al. Enhanced biocompatibility of CD47-functionalized vascular stents. Biomaterials 87, 82-92 (2016).

46. Yang, Z. et al. Nitric oxide producing coating mimicking endothelium function for multifunctional vascular stents. Biomaterials 63, 80-92 (2015).

47. Zhang, H., Ren, K. F., Chang, H., Wang, J. L. \& Ji, J. Surface-mediated transfection of a pDNA vector encoding short hairpin RNA to downregulate TGF-beta1 expression for the prevention of in-stent restenosis. Biomaterials 116, 95-105 (2017).

48. Sweeney, C. J. et al. The antiangiogenic property of docetaxel is synergistic with a recombinant humanized monoclonal antibody against vascular endothelial growth factor or 2-methoxyestradiol but antagonized by endothelial growth factors. Cancer Res. 61, 3369-3372 (2001).

49. Nakano, T., Goto, K. \& Wakabayashi, I. Docetaxel inhibits cyclooxygenase-2 induction in vascular smooth muscle cells. Eur. J. Pharmacol. 654, 150-154 (2011).

50. Bierhalz, A. \& Moraes, Â. Composite membranes of alginate and chitosan reinforced with cotton or linen fibers incorporating epidermal growth factor. Mat. Sci. Eng. A-Struc. 76, 287-294 (2017).

51. Lee, S. et al. Enhanced therapeutic neovascularization by CD31-expressing cells and embryonic stem cell-derived endothelial cells engineered with chitosan hydrogel containing VEGF-releasing microtubes. Biomaterials 63, 158-167 (2015).

52. Heldman, A. W. et al. Paclitaxel stent coating inhibits neointimal hyperplasia at 4 weeks in a porcine model of coronary restenosis. Circulation 103, 2289-2295 (2001).

53. Pan, C. J., Tang, J. J., Weng, Y. J., Wang, J. \& Huang, N. Preparation, characterization and anticoagulation of curcumin-eluting controlled biodegradable coating stents. J. Control Release 116, 42-49 (2006).

54. Liu, S. J., Chiang, F. J., Hsiao, C. Y., Kau, Y. C. \& Liu, K. S. Fabrication of balloonexpandable self-lock drug-eluting polycaprolactone stents using microinjection molding and spray coating techniques. Ann. Biomed. Eng. 38, 3185-3194 (2010) 
55. Hossfeld, S. et al. Bioactive coronary stent coating based on layer-by-layer technology for sirna release. Acta Biomater. 9, 6741-6752 (2013).

56. Song, C. L. et al. Study of novel coating strategy for coronary stents: simutaneous coating of vegf and anti- CD34 antibody. Rev. Bras. Cir. Cardiovasc. 30 159-163 (2015).

57. Wang G. X., Du R. L., Yin T. Y., Wang Y. Z. \& Rao Q. CN patent 103566414 B (2016).

58. Zamani, M., Prabhakaran, M. P. \& Ramakrishna, S. Advances in drug delivery via electrospun and electrosprayed nanomaterials. Int J. Nanomed. 8, 2997-2307 (2013).

59. O'Brien, B., Zafar, H., Ibrahim, A., Zafar, J. \& Sharif, F. Coronary stent materials and coatings: a technology and performance update. Ann. Biomed. Eng. $\mathbf{4 4}$ $1-13$ (2015)

60. Kolandaivelu, K. et al. Ultra-hydrophilic stent platforms promote early vascular healing and minimise late tissue response: a potential alternative to secondgeneration drug-eluting stents. Eurointervention 12, 17 (2016).
61. Lee, Y. H., Mei, F., Bai, M. Y., Zhao, S. \& Chen, D. R. Release profile characteristics of biodegradable-polymer-coated drug particles fabricated by dual-capillary electrospray. J. Control Release 145, 58-65 (2010).

62. Mcginty, S. \& Pontrelli, G. A general model of coupled drug release and tissue absorption for drug delivery devices. J. Control Release 217, 327-336 (2015).

63. Riegger, J. et al. Histopathological evaluation of thrombus in patients presenting with stent thrombosis. a multicenter european study: a report of the prevention of late stent thrombosis by an interdisciplinary global european effort consortium. Eur. Heart J. 37, 1538-1549 (2015).

64. Jang, S., Lim, K. S., Song, J. E. \& Jeong, M. H. Effect of non-polymer titanium dioxide thin film coated stent with heparin in porcine coronary restenosis model. J. Am. Coll. Cardioy 67, 606 (2016).

65. Ma, X. et al. Delayed re-endothelialization with rapamycin-coated stents is rescued by the addition of a glycogen synthase kinase-3beta inhibitor. Cardiovasc Res 86, 338-345 (2010). 